\title{
BIOMARKERS AS EARLY DETECTION TOOL FOR SILICA DUST EXPOSURE-INDUCED HEALTH PROBLEMS
}

\section{KUSMIYATI ${ }^{1}$}

Health Polytechnic Ministry of Health Kupang, Indonesia

Email : kus1979@yahoo.com

\section{ABSTRACT}

Keywords

The silica dust is widely distributed on earth's surface resulting from various activities. This mineral may result in lung damage initially presenting changes of many compounds in biological systems. Silica exposure on lung tissues triggers reactive oxygen species (ROS) production, influences endogenous antioxidant enzymes (superoxide dismutase, catalase and glutathione peroxidase), and release of inflammatory mediators (IL-1, IL-6, TNF- $\alpha$ ). These biomarkers are developed as marker of silica exposure effect in the body. It is concluded that development of biomarkers is warranted for early detection of silica exposure-induced health problems in order to prevent its

\section{Antioxidant; biomarker; detection; inflammatory; reactive oxygen species; silica} progression into serious diseases. 


\section{INTRODUCTION}

Silica is the most abundant mineral on the earth's surface. Silica dust could be found in material of foundry, road construction, glass, cement, coal, and ceramic industries (IAPA 2008). The workers at such places and communities around the location are potential group to develop silica exposure impacts. Every exposure of environmental substances that get into the body may give rise to health impacts. Health impacts that occur in every person are different for it is influenced by contaminant factors (type, size, toxicity, duration of exposure) and individual host factors (susceptibility, age, sex, nutritional status). The daily amount of inhaled air may vary depending on body size, metabolic rate and physical activity (Wallace 2008).

Silicosis has been a historically important occupational disease and continues to be a concern. Silicosis is a fibrotic lung disease due to the inhalation of respirable silica. Workers who may be exposed to high concentrations of free crystalline silica in unprotected settings may be at risk for developing pulmonary fibrosis. Biological marker (biomarker) is indicator of events in a biological system. When an exposure referring to the contact between a substance and surface of the human body through inhalation, ingestion and dermal communication, biomarkers give information about the activity of a substance after its absorption. Biological monitoring provides useful information about exposure, early detection of the effect on health, and susceptibility (Booth \& Stoia 2013).

Silica exposure may lead to lung fibrosis through fibrogenesis process, which is a dynamic process that occurs in four common phases including initiation due to injury, inflammation and activation of effector cells, enhanced synthesis of extracellular matrix, and deposition of extracellular matrix with progression to end-organ failure (Richter et al. 2015). The main mechanisms of disease progression caused by silica-induced lung damage are: direct cytotoxicity, production of reactive oxygen species (ROS) and reactive nitrogen species (RNS), secretion of inflammatory and fibrotic mediators, lung remodeling and cell death by apoptosis (Bernardo et al. 2016). This paper aims to review studies on biomarker changes due to silica exposure.

\section{METHODS}

This review focuses on biomarker dan silica dust. We reviewed English published studies in google scholar and science direct concerning biomarker effects of silica dust exposure as of March 2018 with the following keywords: silica, reactive oxygen species, inflammatory and antioxidant. All published, human studies, experimental animal in vivo and in vitro studies were 
considered for inclusion. All English published studies were included.

\section{RESULTS AND DISCUSSION}

\section{Reactive Oxygen Species}

Reactive oxygen species (ROS) are derivatives of oxygen that are more reactive than molecular oxygen. It was originally thought that only phagocytic cells were responsible for ROS production as their part in host cell defense mechanisms. Recent work has demonstrated that ROS have a role in cell signaling of many biological systems. Generation of ROS in high levels may harm the adjacent tissues. Oxidative stress, arising as a result of an imbalance between ROS production and capability of natural protection mechanism of organism (endogenous and exogenous antioxidants) against reactive metabolites and in preventing any harmful effects. Many substances may be used as biomarkers of oxidative stress such as lipid oxidation, protein oxidation, DNA oxidation, antioxidant capacity, and other parameters.

Oxidants generation by silica particles and by silica-activated cells results in the increased lipid peroxidation, cell and lung injury (Pandey \& Agarwal 2012). Oxidants are generated directly from the reactive surface of crystalline silica and as a result of phagocytosis of silica particles. These reactive oxygen species activate transcription factors controlling message levels in macrophages which induce the production of chemokines, inflammatory cytokines, and fibrogenic factors (Castranova 2004). Low levels of ROS production can result in cell proliferation and activation of antioxidant defenses, but higher ROS levels trigger DNA damage, p53 activation, cell cycle blockade, and cell death via apoptosis and/or necrosis. All of these may be important in a culminating fibrotic response (Cheresh et al. 2013).

Decreased expression of antioxidant enzymes can cause oxidative stress as a result of inability of body to neutralize ROS. Likewise, excessive oxidative stress, in particular from ${ }^{*} \mathrm{OH}$, may cause damage to DNA, proteins, and membrane lipids (Øvrevik et al. 2015) The study found that exposure of intranasal instillation of silica to Sprague dawley rats had increased MDA level in lung tissue (Abdelaziz et al. 2016). Administration of intratracheal silica to female Balb/c mice also elevated level of $\mathrm{H}_{2} \mathrm{O}_{2}$ serum (Sato et al. 2015). Plasma MDA levels of foundry workers exposed to metal dust containing silica were higher than that of in nonexposed group (Liu et al. 2009).

\section{Antioxidant Enzymes}

Endogenous antioxidant enzymes may be used as biomarkers of silica exposure. Silica exposure can trigger ROS 
formation that lead to oxidative stress. Oxidative stress is defined as a disturbance in the balance between production of ROS/RNS and antioxidant defence. Body gives responses as a defense mechanism against ROS. Production of superoxide dismutase enzymes may increase when the production of free radicals are increased in the body to maintain the balance between them. However, if free radicals we produce are increasing that antioxidant in the body cannot compensate, consequently oxidative stress occurs. In addition to trigger expression of antioxidant enzymes, silica exposure also inhibits antioxidant enzyme activity in the body.

A study found that among sandblasting workers, superoxide dismutase activity of patients with silicosis was higher compared to non-exposed control (Palabiyik et al. 2013). The function of superoxide dismutase is to convert peroxide radicals into $\mathrm{H}_{2} \mathrm{O}_{2}$. Silica exposure has been previously demonstrated to cause increased expression and activity of enzymatic antioxidants. A variety of protective mechanisms have evolved to scavenge ROS and protect cells from ROS adverse effects, which are collectively known as the cell's antioxidant defense. The defense mechanisms include vitamins $\mathrm{C}$ and $\mathrm{E}$, coenzyme Q10 and glutathione, as well as antioxidant enzymes, such as glutathione peroxidase (GPx), catalase, and superoxide dismutase (SOD) (Appelqvist 2010).
Silica could impair antioxidant system in the body (Andre et al. 2013). The finding is similar to other study which exposed intranasal instillation of silica to Sprague dawley rats. Silica exposure led to declined lung SOD and GSH level as antioxidant enzymes (Abdelaziz et al. 2016). Various changes of the molecule in the serum and tissue become a marker of early damage in the body before causing visible disease.

\section{INFLAMMATORY MEDIATOR}

Alveolar macrophages play role in development of silicosis by releasing mediators including fibrotic factors, lysosomal enzymes, ROS, cytokines and chemokines interacting in complex tissues to affect lung injury, inflammation and fibrosis. TNF- $\alpha$ and IL-1 have been thought as biomarkers of silicosis (Tiwari 2005). The IL-1 is a key mediator of the host response to various immunologic challenges and infectious inflammatory. IL-6 play a role as mediator of inflammation and silica-induced lung injury too (Sharan et al. 2010). A study using mouse model showed significantly higher levels of IL-6 among silica-treated mice (Freire et al. 2013).

Silica exposure triggers inflammatory cytokines and growth factors, infiltration of inflammatory cells as well as elevates signs of lung fibrosis (Sharan et al. 
2010). Silica and ROS production during phagocytosis may activate transcription factor of NF-kB. In most unstimulated cells, NF- $\kappa \mathrm{B}$ dimers are retained in an inactive form in the cytosol through their interaction with inhibitor of NF- $\mathrm{kB}$ proteins (IкB)(Oeckinghaus \& Ghosh 2009). Secretion of inflammatory mediators by inflammatory cells leads to recruitment of more inflammatory cells into the injury site. Inflammation is a complex response mediated by immune cells, including monocytes, macrophages and others. Inflammation aims to get rid of the causative agent, but if it persists it can cause tissue damage. Exposure of intranasal instillation of silica led to increased lymphocyte count in lung tissues (Abdelaziz et al. 2016).

\section{CONCLUSIONS}

Many studies have been done to develop biomarkers of silica exposure. Further studies are highly warranted to identify the most effective and affordable biomarkers. Therefore, health problems may be early detected and preventive actions could be done as early as possible to prevent its progression into serious diseases in comunity.

\section{REFERENCES}

Abdelaziz, R.R., Elkashef, W.F. \& Said, E., 2016. Tadalafil Reduces Airway Hyperactivity and Protects Against Lung and Respiratory Airways Dysfunction in a Rat Model of Silicosis. International Immunopharmacology, 40, pp.530541.

Andre, B. et al., 2013. Serum selenium and selenoprotein $\mathrm{P}$ in patients with silicosis. Journal of Trace Elements in Medicine and Biology, 27, pp.40-44. Appelqvist, A.J.H., 2010. Regulation of apoptosis-associated lysosomal membrane Permeabilization Regulation of apoptosis-associated lysosomal membrane permeabilization. Apoptosis, 15(May 2014), pp.527-540.

Bernardo, E. et al., 2016. Cell-Based Therapy for Silicosis Cell-Based Therapy for Silicosis. Stem Cells International, 2016(March), p.9.

Booth, K.A. \& Stoia, 2013. Anatomy, Physiology and Disease for The Health Professions, Cina: Mc GrawHill Companies.

Castranova, V., 2004. Signaling Pathways Controlling. Free Radical Biology and Medicine, 37(7), pp.916-925.

Cheresh, P. et al., 2013. Oxidative Stress and Pulmonary Fibrosis. Biochim Biophys Acta, 1832(7), pp.1028-1040. 
Freire, J. et al., 2013. Silica-induced

Chronic Inflammation Promotes Lung Carcinogenesis in the Context of an Immunosuppressive. Neoplasia, 15(8), p.913-924, IN15-IN18.

Available at:

http://dx.doi.org/10.1593/neo.13310.

IAPA, 2008. Silica in The Workplace, Industrial Acciden Prevention Association.

Liu, H. et al., 2009. Health risk assessment by measuring plasma malondialdehyde ( MDA ), urinary 8hydroxydeoxyguanosine ( $8-\mathrm{OH}-\mathrm{dG}$ ) and DNA strand breakage following metal exposure in foundry workers. Journal of Hazardous Matterials, 170, pp.699-704.

Oeckinghaus, A. \& Ghosh, S., 2009. The NF- k B Family of Transcription Factors and Its Regulation. Cold Spring Harb Perspect Biol, 1, pp.115.

Øvrevik, J. et al., 2015. Activation of Proinflammatory Responses in Cells of the Airway Mucosa by Particulate Matter: Oxidant- and Non-OxidantMediated Triggering Mechanisms. Biomolecules, 5, pp.1399-1440.

Palabiyik, S.S. et al., 2013.
Immunomodulation and Oxidative Stress Caused by Silicosis. Arh Hig Rada Toksikol, 64, pp.431-437.

Pandey, J.K. \& Agarwal, D., 2012. Biomarker: A Potensial Prognostic Tool for Silicosis. Indian Journal and Environmental Medicine, 16(3), pp.101-107.

Richter, K. et al., 2015. Redox- fi brosis : Impact of TGF $\beta 1$ on ROS generators , mediators and functional consequences. Redox Biology, 6, pp.344-352.

Sato, T. et al., 2015. Suppressive Oligodeoxynucleotides Inhibit SilicaInduced Pulmonary Inflammation. $J$ Immunol, 180, pp.7648-7654.

Sharan, S. et al., 2010. Pulmonary Fibrosis IL-6 receptor-mediated lung Th2 cytokine networking in silica-induced pulmonary Fibrosis. Arch Toxicol, 84(December), pp.947-955.

Tiwari, 2005. Biomarkers of Silicosis: Potential Candidates. Indian Journal of Occupational and Environmental Medicine, 9(3), pp.103-106.

Wallace, R., 2008. Public Health and Preventive Medicine fifteenth., USA: Mc Graw Hill Companies, Inc. 